in starved SF indicated an important role for PI3K-mediated signals in the ACPA-induced increase of SF mobility.

Conclusions: We demonstrated that additional stimuli (such as stress-induced citrullination and cytokine priming) were needed for SF to react upon ACPA stimulation. This is an indirect proof supporting the idea that a synovial insult that will normally resolve unobserved, might be essential for the transition towards chronic synovial changes in the presence of ACPA.

Disclosure of Interest: None declared

DOI: 10.1136/annrheumdis-2017-eular.6448

\section{FRI0014 ANTIOXIDANT ROLE OF MICROVESICLES FROM ADIPOSE TISSUE-DERIVED MESENCHYMAL STEM CELLS IN HUMAN OSTEOARTHRITIC CHONDROCYTES}

M. Tofiño-Vian ${ }^{1}$, I. Guillen ${ }^{1,2}$, M.D. Perez del $\mathrm{Caz}^{3}$, A. Silvestre ${ }^{4}$, M.J. Alcaraz ${ }^{1}$ ${ }^{1}$ Pharmacology and IDM, University of Valencia, Burjasot, Valencia; ${ }^{2}$ Dpt. Pharmacy, CEU-Cardenal Herrera; ${ }^{3}$ Department of Burn and Plastic Surgery, la Fe University Hospital; ${ }^{4}$ Orthopaedic Surgery and Traumatology, Clinical University Hospital, Valencia, Spain

Background: Oxidative stress results in the disruption of normal physiologic signaling leading to inflammatory changes, cartilage degradation and osteoarthritis (OA) progression (1). Recent advances have revealed the role of cell-derived microvesicles (MV) as a new mechanism of cell-to-cell communication with potential therapeutic applications. We have shown previously the antiinflammatory effects of human adipose tissue-derived mesenchymal stem cells (AD-MSC) conditioned medium in OA chondrocytes (2).

Objectives: We have isolated the MV fraction from the secretome of AD-MSC to investigate its activity on oxidative stress and inflammation in OA chondrocytes stimulated with interleukin (IL)-1 $\beta$. Furthermore, we have characterized the MV protein content by proteomic analysis.

Methods: AD-MSC were isolated from fat of patients who undergone abdominoplasty (without obesity). MV were isolated from AD-MSC conditioned medium by differential centrifugation with size filtration. MV size and concentration were determined by resistive pulse sensing. Proteomic analysis was performed by LC-MS/MS, with ProteinPilot and PeakView software and the bioinformatic tools UNIPROT and PANTHER. OA chondrocytes were isolated from knee specimens of advanced OA patients, stimulated with IL-1 $\beta(10 \mathrm{ng} / \mathrm{mL})$ and treated with MV (3.6 $\times 10^{7}$ particles $/ \mathrm{mL}$ ) for $24 \mathrm{~h}$. Accumulation of 4-hydroxy-2-nonenal (HNE)modified proteins and cytokines were measured by ELISA, NO production and MMP activity by fluorometry. Expression of specific proteins was evaluated by confocal microscopy or immunostaining. The data were analysed by ANOVA followed by Dunnett's test.

Results: MV reduced the accumulation of HNE-modified proteins, a biomarker of oxidative stress-induced lipid peroxidation, in OA chondrocytes stimulated with IL- $1 \beta$. The production of NO, IL- 6 and TNF $\alpha$, as well as MMP activity were also significantly reduced by MV treatment, whereas IL-10 and collagen II were enhanced. Proteomic analysis of MV showed high levels (5.89-fold upregulation) of peroxiredoxin 6 ( $\operatorname{Prdx} 6)$, a member of the peroxiredoxin family of antioxidant proteins which is downregulated in OA cartilage (3). MV treatment increased the expression of Prdx6 in OA chondrocytes suggesting a protective role against oxidative stress in these cells.

Conclusions: MV from AD-MSC downregulate the production of oxidative stress and inflammatory mediators in OA chondrocytes. Prdx6, an antioxidant enzyme, may contribute to the protective effects of MV. Our data support the interest of these MV to develop new therapeutic approaches.

\section{References:}

[1] Loeser RF et al. (2002) Detection of nitrotyrosine in aging and osteoarthritic cartilage: Correlation of oxidative damage with the presence of interleukin1 beta and with chondrocyte resistance to insulin-like growth factor 1 . Arthritis Rheum 46: 2349-2357.

[2] Platas J et al. (2016) Paracrine effects of human adipose-derived mesenchymal stem cells in inflammatory stress-induced senescence features of osteoarthritic chondrocytes. Aging 8: 1703-17.

[3] Ikeda D et al. (2013) iTRAQ-based proteomics reveals novel biomarkers of osteoarthritis. Biomarkers 18: 565-572.

Acknowledgements: Funded by SAF2013-48724-R (MINECO, FEDER) and PROMETEOII/2014/071 (Generalitat Valenciana).

Disclosure of Interest: None declared

DOI: 10.1136/annrheumdis-2017-eular.3682

\section{FRI0015 MODELLING THE INITIAL PHASE OF FRACTURE HEALING IN VITRO - 3D BONE-LIKE MODELS OF ENDOCHONDRAL OSSIFICATION}

M. Pfeiffenberger ${ }^{1}$, A. Lang ${ }^{1}$, I. Ponomarev ${ }^{2}$, D. Barnewitz ${ }^{2}$, F. Buttgereit ${ }^{1}$, T. Gaber ${ }^{1} \cdot{ }^{1}$ Charité University Hospital, Berlin, Germany, Berlin; ${ }^{2}$ Research Center of Medical Technology and Biotechnology, Bad Langensalza, Germany

Background: Immunosuppressed patients with ongoing inflammation experience more often difficulties in the process of fracture healing. Hereby amongst others immunomodulated activation of osteoclasts leads to augmented osteolysis. Most notably numerous cytokines and invading lymphocytes provide an inflammatory environment within the fracture gap, utmost during the first phase of fracture healing. Nowadays researchers, whilst investigating fracture healing, use small rodent models, facing the problem of translation towards the human system. Hence, there is a lack of valid in vitro models to examine the first phase of fracture healing. To test new therapeutic strategies, we develop a valid 3Dmodel using human cells to mimic the first phase of fracture healing in vitro which is characterized by the formation of a fracture hematoma, a hypoxic microenvironment as well as inflammation that initiate the healing cascade and the process of endochondral ossification.

Objectives: To develop 3D bone-like models displaying endochondral ossification. Methods: As a first step, we focus on establishing 3D bone-like, matrix-free models consisting of human mesenchymal stromal cells (hMSC). MSC were isolated from bone-marrow samples of patients undergoing total hip replacement and characterized with regard to their typical surface markers as well as their differentiation potential towards osteogenic, adipogenic and chondrogenic lineage. 3D bone-like models were generated from micro-mass culture of hMSC (Research Center of Medical Technology and Biotechnology, Bad Langensalza). After initial maturation, 3D bone-like models were cultured under hypoxic conditions $\left(37^{\circ} \mathrm{C}\right.$, $1 \% \mathrm{O} 2$ ) in osteogenic medium for up to 3 months. In vitro $\mu \mathrm{CT}$ analyses were performed at day 0 , after one and two months focusing on the total volume (TV) and bone volume (BV). Additionally osteogenic-relevant genes/factors (Runx2, SPP1, DIx5, ALP, RANKL, SPI1) as well as exclusion markers (SOX9, PPARg2) were investigated after 3 months of cultivation using qRT-PCR. Finally, we implemented histological/immunohistochemical methods (van Kossa, ALP, Col1 staining).

Results: The 3D bone-like models achieved diameters between 0.5 and 0.7 and a thickness of $0.3 \mathrm{~cm}$. In vitro $\mu \mathrm{Ct}$ analysis revealed a high amount of mineralized tissue at day 0 and substantial increase in the bone volume (BV/TV) after cultivation. At day 0 in vitro $\mu \mathrm{Ct}$ analysis implied mineralization mostly at the margin but penetrated the tissue within further cultivation. These results are also supported by positive van Kossa staining. Additionally, GRT-PCR results yielded higher expressions of RUNX2, SPP1, SPI1, RANKL and DLx5. Furthermore, immunohistochemistry showed high ALP-activity and Coll-expression.

Conclusions: Preliminary results of our study focusing at developing a 3D bone-like model displayed a promising trend towards modelling endochondral ossification in vitro by increased mineralization (in vitro $\mu \mathrm{Ct}$ analysis and van Kossa staining), and upregulation of osteogenic-relevant RUNX2 and SPP1 expression as well as ALP-activity and Coll-expression. High expression of SPI1 and RANKL could refer to osteoclast-like activities, which will be in the focus of further investigations. Finally, the complete 3D model will leave us the opportunity for studying the first phase of fracture healing under in vitro conditions.

Disclosure of Interest: None declared

DOI: 10.1136/annrheumdis-2017-eular.5899

\section{FRI0016 INVOLVEMENT OF RUNX-2 AND $\beta$-CATENIN SIGNALING IN THE PRODUCTION OF ADAMTS-7 AND ADAMTS-12 IN OSTEOARTHRITIC SYNOVIAL FIBROBLASTS}

S. Pérez-García ${ }^{1}$, I. Gutiérrez-Cañas ${ }^{1}$, R. Villanueva-Romero ${ }^{1}$, J. Leceta ${ }^{1}$, J. Fernández ${ }^{2}$, I. González-Álvaro ${ }^{3}$, Y. Juarranz ${ }^{1}$, R.P. Gomariz ${ }^{1} .{ }^{1}$ Cell Biology, Faculty of Biology, Complutense University of Madrid; ${ }^{2}$ Traumatology Service;

${ }^{3}$ Rheumatology Service, la Princesa University Hospital, Princesa Medical Research Institute, Madrid, Spain

Background: Proteinases released from synovial membrane of osteoarthritis $(\mathrm{OA})$ patients contribute to cartilage damage. We recently reported in synovial fibroblasts (SF) the expression of ADAMTS (a disintegrin and metalloproteinase domain with thrombospondin motifs) -7 and -12 , involved in the destruction of the cartilage oligomeric matrix protein (COMP) (1). Signaling pathways regulating these ADAMTS are poorly understood. As Runx2 and $\beta$-catenin are two transcription factors involved in chondrocytes metabolism and OA pathology (25), we studied whether these factors are implicated in the production of ADAMTS-7 and 12 in SF. Moreover, we analyzed the induction by two inflammatory mediators present in OA joints: interleukin-1 $\beta$ (IL-1 $\beta$ ), and fibronectin fragments (Fn-fs), previously described in ADAMTS expression (1).

Objectives: To elucidate the signaling pathways involved in the production of ADAMTS-7 and -12 in healthy donors (HD) - and OA-SF.

Methods: ADAMTS-7 and -12 were detected in HD- and OA-SF protein extracts by Western blot. Blockade experiments were performed after stimulation with IL-1 $\beta$ or $45-\mathrm{kDa}$ Fn-fs. We used inhibitors for two mitogen-activated protein kinases (MAPKs), ERK and p38, implicated in the activation of Runx2, PD98059 and SB203580, respectively. We also used an inhibitor of $\mathrm{Wnt} / \beta$-catenin signaling, DDK-1. Levels of ADAMTS-7 and -12 were analyzed by quantitative RT-PCR and ELISA in SF culture supernatants.

Results: Intracellular presence of ADAMTS-7 and -12 was confirmed in HD- and OA-SF, with higher levels of ADAMTS-7 in OA. After IL-1 $\beta$ or Fn-Fs stimulation, DDK-1 decreased ADAMTS-7 transcript in HD and OA-SF that was translated to a protein reduction in OA. Besides, ERK inhibitor decreased ADAMTS-12 mRNA and protein exclusively in OA-SF.

Conclusions: We reported that ADAMTS-7 protein expression is higher in OA-SF compared to HD confirming previous data at mRNA level (1). As DKK decreased ADAMTS-7, Wnt- $\beta$-catenin signaling seems to be implicated in its expression. By contrast, the expression of ADAMTS-12 is regulated by ERK, pointing to a possible implication of ERK-Runx2 axis, exclusively in OA-SF. 